\title{
New Granulocyte Antigens Demonstrated by Microgranulocytotoxicity Assay
}

\author{
J. S. Thompson, V. L. Overlin, J. M. Herbick, C. D. Severson, F. H. J. Claas, \\ J. D. 'Amaro, C. P. Burns, R. G. Strauss, and J. A. Koepke, Departments of \\ Medicine, Pediatrics and Pathology, Granulocyte Transfusion Service, \\ University of Iowa and Veterans Administration Medical Center, Iowa City, \\ Iowa; and the Department of Immunohaematology, University Hospital, \\ Leiden, Netherlands
}

A B S T R A C T Although granulocyte transfusions and bone marrow transplantation are becoming common clinical modalities, our knowledge of surface nonerythroid, nonlymphoid, non-HLA hematopoetic antigens remains very incomplete. Accordingly, we have systematically screened sera from recipients of multiple granulocyte and whole blood transfusions, and immunoneutropenic patients for antibodies directed primarily at granulocytes.

The initial screens demonstrated that $>50 \%$ of the sera from the above sources contained non-HLA cytotoxic and/or agglutinating antibodies. Preliminary clustering indicated seven possible new specificities detected by microgranulocytotoxicity. Calculations for Hardy-Weinberg goodness of fit based on a study of 98 unrelated donors plus informative families established that 5 of these were alleles of a single new locus termed Human Granulocyte Antigen (HGA)-3a, b, c, d, and e. Absorptions indicated that these antigens were present on mature granulocytes but absent from platelets, lymphocytes, monocytes, and myeloid precursors. A single antigen of another separate locus, HGA-1, was also identified. Absorptions revealed a quite different distribution for HGA-1 than HGA-3, this antigen being detected on monocytes and myeloblasts as well as on mature granulocytes. Independent segregation of the three loci from HLA, from the NA-NB and the 5a-5b antigens, and from themselves was confirmed in informative families.

Finally, it seems likely that other antigens will be identified because several other sera that react with both monocytes and granulocytes have been detected.

Received for publication 6 August 1979 and in revised form 28 January 1980.

\section{INTRODUCTION}

Recent widespread use of granulocyte transfusions to support patients with severe chemotherapy-induced neutropenia, and the sharp increase in bone marrow transplantation have reawakened interest in the surface antigens on granulocytes and their precursors. Substantial evidence exists that the concentration of HLA-A and HLA-B antigens on granulocytes is considerably less than on lymphocytes $(1,2)$. Despite a number of investigations, no beneficial or harmful effect of the transfusion of HLA mismatched granulocytes into sensitized recipients has been conclusively demonstrated (3-5).

Granulocytes possess unique antigens that have been demonstrated primarily by leukoagglutination tests, i.e., $\mathrm{NA}_{1}, \mathrm{NA}_{2}$, and $\mathrm{NB}_{1}(6,7) 9 \mathrm{a}(8)$ and more recently by both agglutination and indirect immunofluorescence, i.e., $\mathrm{ND}_{1}(9)$ and $\mathrm{NE}_{1}(10)$. Although many of the antisera detecting these specificities have been obtained from patients suffering from neonatal or acquired immunoneutropenia, there has also been no report to date that mismatching for human granulocyte antigens has been a negative factor in either granulocyte transfusion therapy or in bone marrow transplantation $(3-5,11)$. Therefore, we have set out to systematically search for additional antigens involving granulocytes that may serve as clinically important determinants in these and other conditions. Recently, we have provisionally reported evidence for more than one new granulocyte system detected by a double fluorochromatic microgranulocytotoxic assay $(12,13)$.

This report extends those observations and describes a new polymorphic locus with genes coding for antigens that are confined to mature granulocytes with at least five alleles. These studies also provide evidence for 
other antigens that are shared by granulocytes and monocytes.

\section{METHODS}

Granulocytotoxicity (GCY). ${ }^{1}$ The double fluorochromatic microgranulocytotoxicity method has been previously reported in detail (14). Minor modifications at one of the centers (Leiden) involved substitutions of 5\% Dextran (200,000 mol wt, Poviet, Amsterdam) in phosphate-buffered saline for $2 \%$ methylcellulose-15 (278, Fisher Scientific Co., Pittsburgh, Pa.) for erythrocyte sedimentation; $1 \%$ bovine serum albumin in phosphate-buffered saline in place of tris-buffered saline $(0.9 \%$ $\mathrm{NaCl}$ buffered with $0.001 \mathrm{M}$ Tris- $\mathrm{HCl}, \mathrm{pH} 7.2$ ) as the standard washing and diluent solution; and the substitution of 0.025 $\mu \mathrm{g} / \mathrm{ml}$ carboxyfluorescein diacetate (kindly provided by Dr. J. W. Bruning, Leiden) for diacetylfluorescein (1688, Eastman Organic Chemicals Div. Eastman Kodak Co., Rochester, N. Y.) as the fluorochromatic label for viable granulocytes. None of these substitutions significantly altered the cell preparation except that the carboxyfluorescein diacetate provided a more stable fluorescent label.

After incubation of $1.0-\mu$ l cells $\left(\sim 3.0-4.0 \times 10^{6} / \mathrm{ml}\right)$ with $1.0 \mu \mathrm{l}$ antisera under oil in tissue typing trays for either $30 \mathrm{~min}$ at $23^{\circ} \mathrm{C}$ or for $60 \mathrm{~min}$ at $20^{\circ} \mathrm{C}$, the wells were flooded with Hanks' balanced salt solution (HBSS), and the excess fluid was gently aspirated. $5 \mu \mathrm{l}$ rabbit complement diluted 1:1.25$1: 1.75$ in HBSS was added and incubated for 70-120 min at $20^{\circ} \mathrm{C}$ (the time varies with the complement titer). The dilution and incubation of complement are critical variables that were controlled by testing a matrix of positive and negative sera against the same cell donors. This analysis was performed with each new lot of complement and on several occasions while establishing the method in Leiden. After complement incubation, the reaction was stopped by the addition of 1.0 $\mu$ l $2.5 \%$ ethylenediaminetetraacetic acid, disodium (EDTA$\mathrm{Na}_{2}$ buffered to pH 7.2 with Tris-HCl) which also contained $0.03 \%$ ethidium bromide to label the nuclei of the complement-mediated cytotoxically injured cells. The reactions were scored $(8=80 \%-100 \%$ dead, $6=60 \%-80 \%, 4=40 \%-60 \%$, $2=10 \%-40 \%, 1=<10 \%)$ using fluorescent microscopes equipped with excitation and barrier filters to allow simultaneous visualizations of green-red fluorescence ( $2 \mathrm{~mm}$ UG- 1 excitation with two, 4 mm BG-38 heat barrier and a K580 suppression filter E. Leitz, Inc., Rockleigh, N. J. LP455-SP490 with an LP520 barrier filter, Carl Zeiss, Inc., New York).

Monocytotoxicity. From the same sample of blood used for GCY, the mononuclear layer was isolated following centrifugation at $1,000 \mathrm{~g}$ for $15 \mathrm{~min}$ through a Ficoll-isopaque (or hypaque) density gradient. The cells were washed twice in $1 \%$ bovine serum albumin-phosphate-buffered saline, suspended in RPMI 1640 with $20 \%$ autologous or pooled cytotoxic-free $\mathrm{AB}$ serum and incubated in $60-\mathrm{mm}$ plastic culture dishes for $30 \mathrm{~min}$ at $37^{\circ} \mathrm{C}$ in humidified $5 \% \mathrm{CO}_{2}$-air. Nonadherent cells were collected. The remaining adherent cells were vigorously washed six times with cold HBSS. Adherent cells were removed with lidocaine as described by Rinehart et al. (15) except that the final concentration of lidocaine was reduced to $26 \mathrm{mM}$ in HBSS, incubation was shortened to 10 min at room temperature, and all washes of the monocyteenriched cells after harvesting them from the culture dishes

${ }^{1}$ Abbreviations used in this paper: CAN, capillary agglutination with neutrophils; GCY, granulocytotoxicity; HBSS, Hanks' balanced salt solution; HGA, human granulocyte antigen; LCY, lymphocytotoxicity. were performed with $0.5 \%$ bovine serum albumin-HBSS chilled to $4^{\circ} \mathrm{C}$. These modifications increased the cell yield, reduced clumping, and improved viability. The percentage of monocytes in the nonadherent and adherent samples was estimated by alphanaphthyl butyrase staining (16). The concentration of monocytes was $86 \pm 4 \%$ in the adherent aliquot and $<0.5 \%$ in the non-adherent aliquot. The performance of the monocytotoxicity and nonadherent lymphocytotoxicity (LCY) tests thereafter was identical to the method for fluorochromatic GCY.

Antisera. Several hundred samples of serum were examined from multigravida females, patients experiencing febrile transfusion reactions, recipients of multiple granulocyte transfusions, and from individuals suffering from either neonatal or acquired immunoneutropenia. The best antisera were generally selected from this prescreening on the basis of tentative clustering and strength of reactivity. Several index sera were tested against panels of 50 or more cells on three occasions to check reproducibility and the validity of the tentative clusters.

Immunoglobulin class. To determine the immunoglobulin (Ig) class of granulocyte antibodies, the technique of antibody neutralization by anti-Ig reagents (17) was performed except that it was not necessary to remove the neutralized Ig-anti-Ig precipitates by ultracentrifugation. After incubation of $1.0 \mu \mathrm{l}$ antiserum with $1.0 \mu \mathrm{l}$ of either goat $\mathrm{F}\left(\mathrm{ab}^{\prime}\right)_{2}$ anti-human IgM, or IgG for $30 \mathrm{~min}$ at $37^{\circ} \mathrm{C}$, known positive or negative cells were added and the cytotoxicity test continued as described above. The Ig class was determined by which anti-Ig reagent caused a positive reaction to become negative.

Cell donors. Normal cell donors completely characterized for HLA-A, - B, -C; $\mathrm{NA}_{1}, \mathrm{NA}_{2}, \mathrm{NB}$; and 5a-5b (antigens independent of HLA present on granulocytes, lymphocytes, and platelets) $(18,19)$ constituted the cell panels at both institutions. The population study involving 98 unrelated normal donors was performed at one institution. In addition, families were tested at both institutions, all of which were characterized for HLA-A, -B, -C, and -DRw and many of which were also typed for $\mathrm{NA}_{1}, \mathrm{NA}_{2}, \mathrm{NB}_{1}$, and $5 \mathrm{a}-5 \mathrm{~b}$. Patients were not included in the family analysis.

Absorptions. Absorptions were performed with isolated granulocytes, T and B, lymphocytes, platelets, myeloblasts from patients with acute myelocytic leukemia, and enriched monocytes. The sera were first titrated to determine the last two-fold dilution giving a strongly positive reaction. One dilution stronger than this endpoint was absorbed for 30 to 60 min at $23^{\circ} \mathrm{C}$ with $5.0-6.0 \times 10^{6}$ cells $/ 100 \mu$ l except for platelets in which case absorptions were performed two times with 0.5 vol of packed platelets to serum.

Statistical methods. Two-by-two contingency tables were prepared from all possible serum pairs and for all serum-antigen combinations. Based on these tables, chi-square $\left(\chi^{2}\right)$ for independence (with Yate's correction) and correlation coefficients $\left(r=\sqrt{\chi^{2} / \mathrm{N}}\right.$ were calculated.

The fit of the observed and expected phenotype combination (which is a test of Hardy-Weinberg equilibrium) were tested for significance using K. Pearson's chi-square formula (20). Gene frequencies (GF) were calculated from the observed phenotype frequency $(\mathrm{PF}): \mathrm{GF}=1-\sqrt{1-\mathrm{PF}}$; expected heterozygosity was calculated by the formula: $E=(G F$ $\left.\times G_{j}\right) \times 2 \times$ the number of cells tested $(n)$, where $i, j$, etc. $=$ antigens being tested; and $\chi_{2}=\sum(\mathrm{O}-\mathrm{E})^{2} / \mathrm{E}$, where $\mathrm{O}$ $=$ observed heterozygosity. In this analysis, the number of degrees of freedom (d.f.) is equal to the number of phenotype classes, minus 1 for the $(-/-)$ combination, minus 1 for each antigen/-combination, and minus 1 for each time that expectations are pooled. 


\section{RESULTS}

Initial antisera screening. In the early phases of this study, sera were collected from many sources and screened for LCY, GCY, and granulocyte agglutinating reactivity. Several hundred sera were examined from 224 patients including sequential samples taken before, during, and after multiple granulocyte transfusion therapy. In addition, sera from 32 normal male and female controls were also analyzed (Table I). Whereas neither agglutinating nor cytotoxic antibodies were commonly present in the control sera, including samples from multiparous females, the incidence rose strikingly in blood-transfusion patients. The highest frequency, however, occurred in patients suffering from idiopathic symptomatic immunoneutropenia and recipients of multiple granulocyte transfusions. Antibodies reactive with granulocytes were detected in over $50 \%$ of these samples, but the incidence of LCY antibodies was low in both groups.

In 10 patients with severe secondary drug-induced neutropenia, no antibodies were detected before the onset of supportive granulocyte transfusions (Table II). Sequential samples during and after these transfusions were obtained and revealed the appearance of granulocyte antibody from 1 to $72 \mathrm{~d}$ after completion of the transfusion therapy. An additional interesting observation is that only one patient of this group developed antilymphocyte antibodies. A typical sequence is illustrated with another patient (MOO) whose sera have proved to be an excellent reagent detecting one of the alleles of a new granulocyte locus to be described below. Although agglutinins were never observed, GCY antibody first appeared at the end of 20 transfusions. The strength of the antibody rose to a titer of $1: 4$ within another $2 \mathrm{wk}$, remained constant for $3 \mathrm{mo}$ and declined in the following $3.5 \mathrm{mo}$. The Ig class responsible for the cytotoxicity was investigated by neutralization with $\mathrm{F}\left(\mathrm{ab}^{\prime}\right)_{2}$ heavy chain-specific anti$\operatorname{IgM}, \operatorname{IgG}(17)$, and found to be IgG in all samples from this patient. These same characteristics with re-

TABLE I

Comparison of GCY, Capillary Agglutination with Neutrophils (CAN), and LCY Reactivity

\begin{tabular}{lcccr}
\hline & $\begin{array}{c}\text { Subjects } \\
\text { tested }\end{array}$ & GCY & CAN & LCY \\
\hline & & $\%$ & $\%$ & $\%$ \\
Normal controls & 32 & 6 & 12 & 0 \\
$\begin{array}{l}\text { Blood transfusions } \\
\quad \text { Without reactions }\end{array}$ & 44 & 29 & 27 & 4 \\
$\quad$ With reactions & 82 & 57 & 42 & 19 \\
Granulocyte transfusions & 38 & 61 & 50 & 16 \\
Immunoneutropenic & 28 & 68 & 57 & 0 \\
\hline
\end{tabular}

TABLE II

Development of Antibody following Completion of Granulocyte Transfusion Therapy

\begin{tabular}{|c|c|c|c|c|}
\hline \multirow[b]{2}{*}{ Patients } & \multirow{2}{*}{$\begin{array}{c}\text { No. } \\
\text { granulocyte } \\
\text { transfusions }\end{array}$} & \multirow{2}{*}{$\begin{array}{l}\text { Sera } \\
\text { tested }\end{array}$} & \multicolumn{2}{|c|}{ Antibody } \\
\hline & & & Onset & Type \\
\hline & & & $d$ & \\
\hline S.O. & 5 & 4 & 1 & GCY-LCY \\
\hline D.W. & 2 & 4 & 6 & CAN \\
\hline T.B. & 6 & 6 & 23 & CAN \\
\hline B.T. & 12 & 6 & 24 & CAN-GCY \\
\hline L.B. & 8 & 6 & 28 & CAN \\
\hline A.L. & 8 & 5 & 49 & GCY \\
\hline R.K. & 22 & 4 & 61 & GCY-CAN \\
\hline J.D. & 4 & 7 & 72 & CAN \\
\hline R.D. & 7 & 6 & 72 & CAN \\
\hline D.T. & 21 & 8 & 17 & CAN \\
\hline
\end{tabular}

spect to induction and Ig class of antibodies to granulocytes have been observed in six other postgranulocyte transfusion sera tested. Granulocyte antibodies obtained from four adult immunoneutropenia patients, however, have been exclusively IgM.

Preliminary clusters. After the initial screening to identify prospective reagents, secondary screens involving 53-67 unrelated normal cell donors were performed to test for provisional clusters. In addition, $2 \times 2$ chi-squares and correlation coefficients for experimental sera, HLA-A, -B, -C; $\mathrm{NA}_{1}, \mathrm{NA}_{2}, \mathrm{NB}$, and $5 a-5 b$ antigenic phenotypes were used to cluster sera with similar reactivity patterns. Several clusters have been identified. The first was defined by three antisera derived from two recipients of multiple granulocyte transfusions (WRI and ROO) and an individual with immunoneutropenia $(\mathrm{CON})$. This cluster has been termed Human Granulocyte Antigen-1 (HGA-1, Table III).

On the other hand, five other provisional clusters have been identified by three or more sera in most cases. The initial observations with three of these clusters revealed not only their internal positive correlations, but also the fact that the correlation coefficients among sera from different clusters were often

TABLE III

Correlations (r) of Sera Distinguishing HGA-1

\begin{tabular}{r|ccc} 
CON & - & & \\
WRI & 0.603 & - & \\
ROO & 0.631 & 0.539 & - \\
\cline { 2 - 4 }$($ Name $)$ & CON & WRI & ROO \\
\hline
\end{tabular}


TABLE IV

Correlations ( $r$ ) of Sera Distinguishing Three Antigenss of the HGA-3 Locus

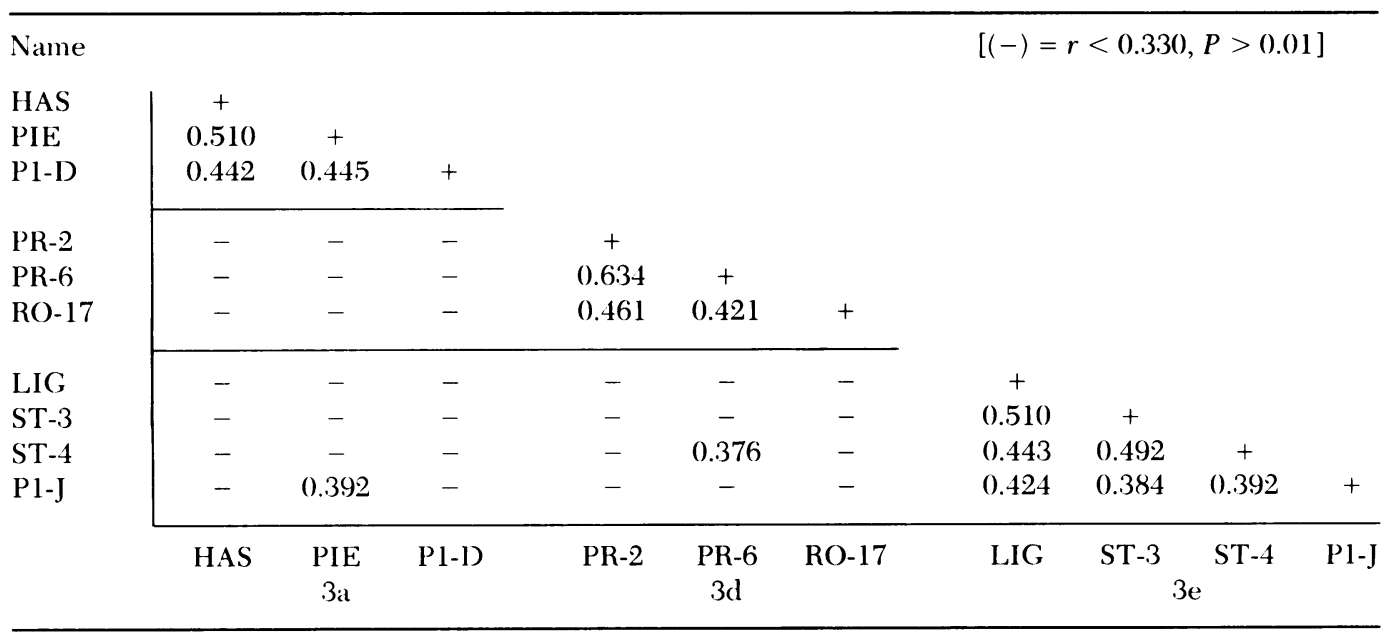

very low or negative, suggesting the possibility that the sera might be reacting against allelic antigenic determinants (Table IV). The other two clusters were represented by three sera and two sera, respectively. Because they were detected during different screens, it was not initially possible to determine their potential relationship to the three clusters shown in Table IV or to HGA-1.

$H G A-3 a, b, c, d, e$. To test for possible allelism vs. independent loci, the best index reagents identifying all of these provisional antigens were included in a third study in which 98 unrelated normal control cell donors were tested. The test results are shown in Fig. 1.

There were five cells with three of the provisional antigenic specificities (triplets) instead of the expected maximum of two specificities for allelic determinants from a single locus. This observation suggests that some of the antisera were not monospecific.

When the five triplet cells were excluded, as is necessary for the calculations, the fit for the HardyWeinberg equilibrium was consistent with the hypothesis that HGA-3 is a multiallelic system at a single genetic locus (Table $\mathrm{V}$ ).

Calculations to test whether HGA-1 was an additional allele of the HGA-3 locus revealed that it was not allelic. Pearson's chi square values of the goodness of expected vs. observed fit of HGA-1 with HGA-3a $=10.80,3 \mathrm{~b}=14.33,3 \mathrm{c}=0.22(P>0.50), 3 \mathrm{~d}=8.21$, and $3 \mathrm{e}=0.23(P>0.50)$. In addition, HGA-1 formed triplets on 12 cells that typed for two HGA-3 antigens, clearly confirming the independence of this antigen from the polymorphic HGA-3 locus.

The distinction of HGA-1 from HGA-3 was further documented by semiquantitative absorption of index sera of these antigens by platelets, $\mathrm{T}$ and $\mathrm{B}$ lymphocytes, monocytes, myeloblasts, and peripheral blood neutrophils (Table VI). For comparison, absorption of two monospecific HLA typing sera are illustrated, HLA-B7, HLA-A1. Whereas $100 \mu \mathrm{l}$ of these sera were absorbed by an equal volume of packed platelets, and by $6 \times 10^{6}$ isolated $\mathrm{T}$ and $\mathrm{B}$ lymphocytes, monocytes, and myeloblasts, this concentration of granulocytes did not absorb their reactivity. HGA-1 sera (WRI, CON), however, were not absorbed by either platelets or T and B lymphocytes but the activity was completely removed by monocytes, myeloblasts, and neutrophils. In contrast, only mature neutrophils were able to

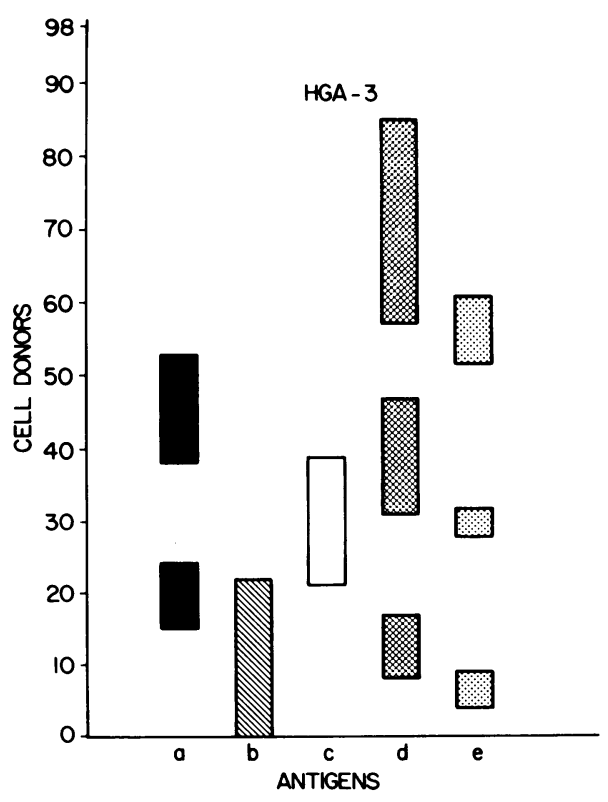

Figure 1 Serogram depicting the reactions of the presumptive $a, b, c, d$, and e alleles of HGA-3 with isolated granulocytes from 98 unrelated normal donors. 
TABLE V

Goodness of Fit Test for Allelism of Five Provisional Granulocyte

Antigens: HGA-3a, $3 b, 3 c, 3 d$, and $3 e$

\begin{tabular}{lccccc}
\hline Antigen & $\begin{array}{c}\text { Gene } \\
\text { frequency* }\end{array}$ & Phenotype & OBS & EXP* & $\frac{(\mathrm{O}-\mathrm{E})^{* *}}{\mathrm{E}}$ \\
\hline HGA-3a & 0.1140 & $\mathrm{a} / \mathrm{b}$ & 4 & $2.547(\mathrm{a})$ & \\
& & $\mathrm{c}$ & 2 & $1.785(\mathrm{a})$ & \\
& & $\mathrm{d}$ & 8 & 6.620 & 0.288 \\
& & $\mathrm{e}$ & 1 & $1.910(\mathrm{a})$ & 0.082 \\
& & - & 5 & 7.133 & 0.638 \\
HGA-3b & 0.1281 & $\mathrm{~b} / \mathrm{c}$ & 0 & $1.881(\mathrm{~b})$ & \\
& & $\mathrm{d}$ & 7 & 6.974 & 0.000 \\
& & $\mathrm{e}$ & 4 & $2.013(\mathrm{~b})$ & \\
HGA-3c & 0.0842 & - & 5 & 7.583 & 0.880 \\
& & $\mathrm{c} / \mathrm{d}$ & 6 & $4.889(\mathrm{~b})$ & \\
HGA-3d & 0.3122 & $\mathrm{e}$ & 3 & $1.411(\mathrm{~b})$ & 0.606 \\
& & $\mathrm{~d} / \mathrm{e}$ & 4 & 5.035 & 0.213 \\
HGA-3e & 0.0901 & $\mathrm{e} /-$ & 4 & 25.289 & 0.290 \\
Null & 0.2794 & - & 12 & 5.437 & 0.066 \\
Totals & & & 93 & 92.999 & 0.380 \\
\hline
\end{tabular}

Pearson's chi-square $=6.536$ ( 5 d.f.); $P=0.257$.

* See text for formulas.

absorb the reactions of two-index HGA-3 sera suggesting that the tissue distribution of HGA-1 and HGA-3 antigens are quite different.

Further evidence for the presence of common antigens on granulocytes and monocytes was provided by testing anti-HGA-1 and HGA-3 reagents and a number of unclassified antisera on granulocytes, monocytes, and lymphocytes isolated from the same sample of peripheral blood (Table VII). Because we did not want to exclude the possibility of detecting new antigens possibly shared by granulocytes, monocytes, and platelets, only unabsorbed sera known to be free of LCY reactivity were selected. Consistent with its absorption, HGA-1 (WRI) reacted strongly with the granulocytes and monocytes from the same cell donors. However, the HGA-3c and HGA-3d sera never reacted

TABLE VI

Semiquantitative Absorption of HLA, HGA-1, and HGA-3 Antisera

\begin{tabular}{|c|c|c|c|c|c|c|}
\hline \multirow{3}{*}{$\begin{array}{l}\text { Absorbing } \\
\text { cells }\end{array}$} & \multicolumn{6}{|c|}{ Antisera } \\
\hline & \multicolumn{2}{|c|}{ HLA- } & \multicolumn{2}{|c|}{ HGA-1 } & \multicolumn{2}{|c|}{ HGA-3 } \\
\hline & Al & B7 & $\mathrm{CON}$ & WRI & PRO & FAN \\
\hline None & $+^{*}$ & + & + & + & + & + \\
\hline Platelets & $-\ddagger$ & - & + & + & + & + \\
\hline \multicolumn{7}{|l|}{ Lymphocytes } \\
\hline$T$ & - & - & + & + & + & + \\
\hline B & - & - & + & + & + & + \\
\hline Monocytes & - & - & - & - & + & + \\
\hline Myeloblasts & - & - & - & - & + & + \\
\hline \multicolumn{7}{|c|}{ Mature polymorphonuclear } \\
\hline lymphocytes & + & + & - & - & - & - \\
\hline
\end{tabular}

* Positive test with informative cells (cytotoxicity scores of 4, 6, or 8).

$\ddagger$ Negative test after absorption. 
TABLE VII

Comparative GCY, Monocytotoxic, and LCY Reactions by HGA, NA, Levamisole-induced, and Unclassified Cytotoxic Antisera

\begin{tabular}{lccc}
\hline \multicolumn{1}{c}{ Antisera } & GCY* & Monocytotoxic & LCY* $^{*}$ \\
\hline HGA-1 (WRI) & $+^{*}$ & $+^{*}$ & $-^{*}$ \\
HGA-3c(SHE) & + & - & - \\
HGA-3d(PRO) & + & - & - \\
NA (LOM) & + & - & - \\
Levamisole & + & + & - \\
Unclassified (AYD) & + & + & - \\
Unclassified (RUG) & + & + & - \\
\hline
\end{tabular}

* Simultaneous reactions on informative cells; a positive $(+)$ reaction $=4,6$, or 8 cytotoxic score tested in duplicate. $\ddagger$ (SER), Abbreviation of serum tested.

with monocytes. LOM is a very interesting serum that detects $\mathrm{NA}_{1}$ by cytotoxicity rather than by agglutination. These reactions were also confined to granulocytes.

On the other hand, several sera from patients obtained during the nadir of Levamisole-induced (Janssen Pharmaceuticals, Beerse, Belgium) agranulocytosis (unpublished observation), a previously wellcharacterized granulocyte-monocyte serum (AYD) and
RUG (a representative of a group of sera that have been obtained from immunoneutropenics and bone marrow transplantation recipients) reacted with both granulocytes and monocytes (Table VII).

There were no significant correlations between the HGA-1 and HGA-3 antigens and the HLA, NA-NB, or $5 a-5 b$ phenotypes of the unrelated cell donors.

Finally, 12 families (both parents plus at least two children) have been studied to determine whether or not the new granulocyte antigens segregate independently of HLA. Six of the families were also typed for $\mathrm{NA}_{1}, \mathrm{NA}_{2}, \mathrm{NB}_{1}$, and $5 \mathrm{a}-5 \mathrm{~b}$ by capillary with isolated granulocytes. All of the typing sera had been included in previous workshops and were considered to be strong monospecific reagents. One of these families (HSF) was highly informative because the father was homozygous for the uncommon $5 \mathrm{a}$ antigen, lacked $\mathrm{NB}_{1}$, and was homozygous for $\mathrm{NA}_{2}$ (Fig. 2). There were four children divided into two male and female HLAidentical pairs, i.e., A2,B5/A2-B15 and A3,B35/A1,B13. One of each pair inherited the maternal HGA-3a antigen. In addition, its inheritance could also be distinguished from maternal $\mathrm{NB}_{1}, 5 \mathrm{a}$, or $5 \mathrm{~b}$. Similarly, there was independent segregation of paternal HGA-3d and HGA-3e from $\mathrm{NB}_{1}$ and $\mathrm{NA}_{2}$ but it was not possible in this family to establish independence of these

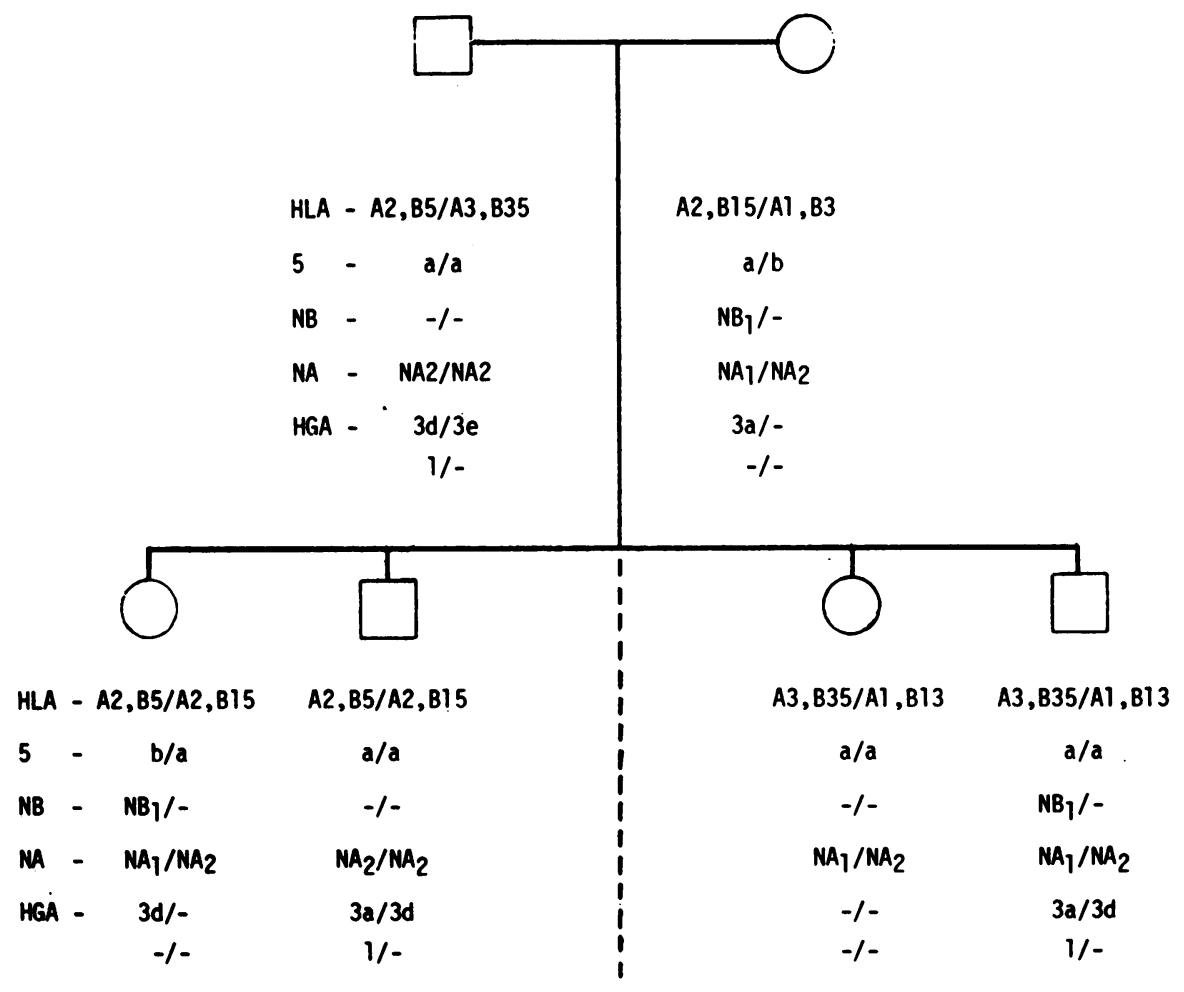

FIGURE 2 Family pedigree, HSF. A(-l-) indicates the presumed segregation of an antigen that was not directly demonstrated but may be inferred from the family study. HLA, 5, NB, NA, and HGA are leukocyte antigenic groups (see text). 
granulocyte antigens from paternal HLA or 5a inheritance. Evidence for independence of HGA-1 from the other antigenic systems was clarified by the facts that one each of the HLA-identical pairs inherited the paternal HGA-1, these two individuals differed for HGA-3d and HGA-3e and, in both cases, HGA-1 caused a triplet to occur.

The second family (RHM) also provided evidence for the independence of HGA-1 from the HGA-3 locus and from HLA (Fig. 3). Again, there were two pairs of HLA identical sibs, i.e., A29,B12/A3,B7 and Aw32,$\mathrm{B} 14 / \mathrm{Al}, \mathrm{B} 8$ plus two other sibs that had inherited the other two alternatives, i.e., Aw32,B14/A3,B7 and $\mathrm{A} 29, \mathrm{~B} 12 / \mathrm{A} 1, \mathrm{~B} 8$. Therefore, it was possible to determine independent segregation of HGA-3a, 3e, and $3 c$ from HLA: paternal HGA-3a was detected in at least one member of each of the HLA identical pairs that differed for their paternal haplotypes; HGA-3e was present in one individual with paternal A29,B12 and in the other with Aw32,B14; similarly, HGA-3c was inherited by sibs with different maternal haplotypes.

This family also confirms the independence of these loci from $\mathrm{NA}_{1}$, in this case detected by GCY. We have previously reported that the cytotoxic but not the agglutinating reactions of a multiparous serum (LOM) react specifically with $\mathrm{NA}_{1}$-positive granulocytes. All children inherited this paternal antigen illustrating its completely independent segregation from the other granulocyte and lymphocyte antigens.

\section{DISCUSSION}

GCY assays have been described previously by Engelfriet (21), Thorsby (22), Hasegawa et al. (23), and Drew et al. (24). Certain problems relative to the innate characteristics of granulocytes to adhere to surfaces, relatively short life span, and presumed unusual complement sensitivity have required specialized techniques to overcome these problems and to produce a reliable reproducible assay. The double fluorochromatic complement-dependent cytotoxicity method reported by Blaschke et al. (14) satisfies these requirements and has proved to be a relatively simple test to establish, with somewhat different reagents, in the Netherlands. It differs in two important technical aspects from the method developed by Drew et al. (24) in that the granulocytes are not treated with papain and no agent to prevent sticking, such as alcohol, is necessary because live green-fluorescing and dead red-fluorescing cells may be visualized throughout the well. Whereas they have reported that optimum cytolysis occurred when antibody was incubated at $5^{\circ} \mathrm{C}$ and that the Ig class detected by the papainized cells was exclusively Ig.M, we have found that incubation temperatures from $20^{\circ}$ to $30^{\circ} \mathrm{C}$ were optimal for the fluorochromatic assay, higher temperatures tended to nonspecifically kill granulocytes, and lower temperatures usually caused lower reactivity. In addition to serum LOM that was previously shown to be $\operatorname{IgG}(25)$, MOO is representative of other reagents that have developed as a consequence of multiple granulocyte transfusions and found to be IgG. This is not exclusive, however, and at least one HGA-3a serum antibody is an IgM protein. The fact that the fluorochromatic GCY method can detect both IgG and IgM antibodies may account for the rather marked difference in the frequency of positive reactions by sera from alloimmunized donors: $<25 \%$ by the papain method $(24)$ as compared with $>50 \%$ by the fluorochromatic technique.

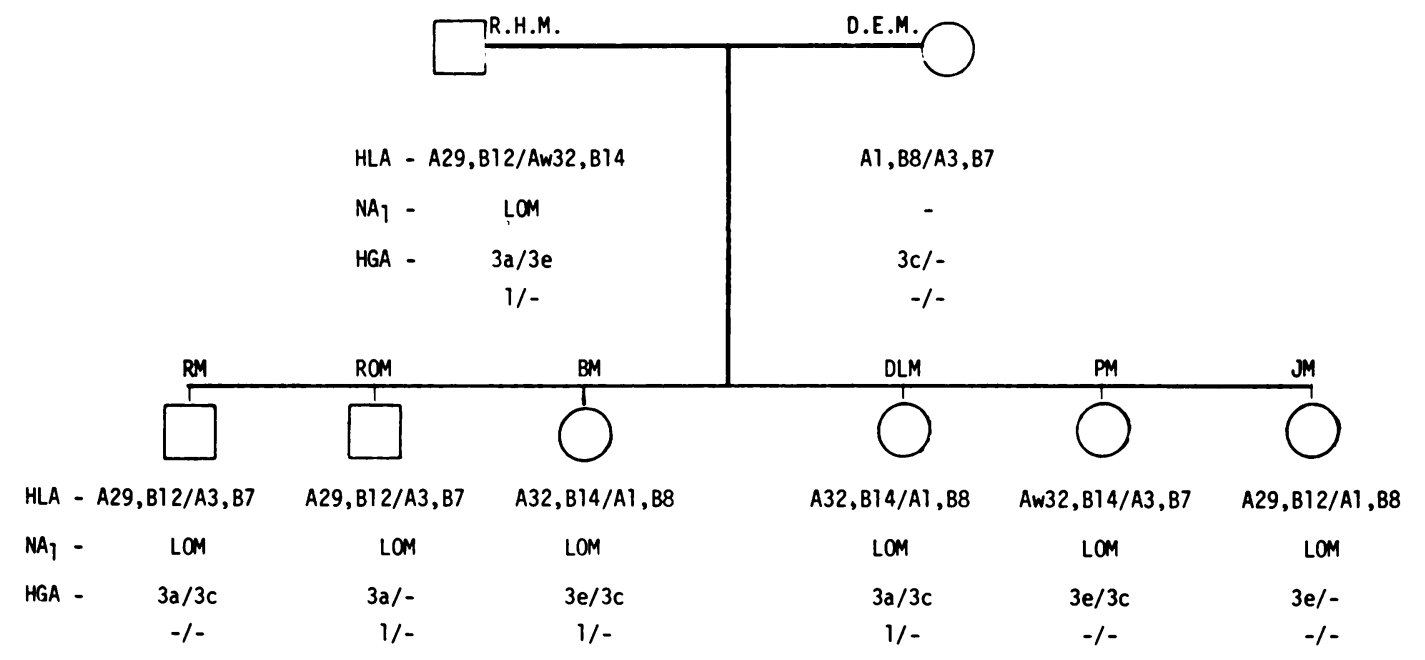

FIgURE 3 Family pedigree, RHM. A(-/-) indicates the presumed segregation of an antigen that was not directly demonstrated but may be inferred from the family study. HLA, 5, NB, NA, and HGA are leukocyte antigenic groups (see text). 
Two aspects of GCY have proven to be a great advantage in demonstrating new granulocyte-specific antigens. Firstly, HLA antisera generally fail to react with mature granulocytes in cytotoxicity testing (14), yet they may specifically react in capillary agglutination (26) and absorption may be accomplished with high concentrations of granulocytes. Secondly, we have demonstrated that the same sera may exhibit quite different reaction patterns with the same granulocyte donors when tested by cytotoxicity as compared with agglutination (25). LOM represents the only serum we have encountered that detects one of the neutrophil-specific NA antigens by cytotoxicity, yet its agglutination reactions are quite different. Thus, it has been possible to screen rather large numbers of sera for GCY without having to first absorb them with platelets or lymphocytes to remove anti-HLA antibodies. This position has been subsequently supported by the observations that GCY reactivity was not absorbed by lymphocytes or platelets.

It was with this background that we undertook the screening of large numbers of antisera in anticipation of finding reagents demonstrating new granulocyte antigens. This report describes a total of seven provisional granulocyte antigens. Of these, the group HGA-3 is particularly interesting because it appears to be the first polymorphic locus whose gene products are present on granulocytes. Recognizing that our sera are roughly comparable in quality to the early HLA reagents, it nevertheless has been possible to distinguish five specificities that satisfy the criteria for allelism in that calculations for goodness of fit were well within the limits of significance. They did not form an unacceptable number of triplets and they segregated as alleles of one locus in informative families. The combined gene frequency of the five HGA-3 specificities is only 0.7231 . This could indicate either that the antisera were too weak to detect all of their potential targets or, more likely, it suggests that other alleles may be found. The family studies support the latter hypothesis because segregation in three informative families, including family RHM, demonstrates that a fourth unidentified allele was present. It also seems possible, in light of the history of HLA serology, that subsequent sera may better define the identified antigens. Family segregation studies document that HGA-3 is not apparently associated with HLA nor with the NA-NB or $5 \mathrm{a}-5 \mathrm{~b}$ antigens. Absorption studies indicated that the HGA-3 antigens were present only on mature granulocytes, making them comparable in that regard to the neutrophil-specific NA-NB antigens $(6,7)$.

Another granulocyte antigen, termed HGA-1, was different in several aspects from HGA-3: goodness of fit calculations and triplet counting in the unrelated population study plus clearly independent segregation in the family studies distinguish it from HGA-3 and this was confirmed by the demonstration of the HGA-1 antigen on both immature and mature granulocytes and on monocytes. Furthermore, parallel HGA-1 reactions were detected on both monocytes and granulocytes from the same cell donors whereas the HGA-3 antisera that have been so tested have reacted only with granulocytes. This may represent one of several granulocyte-monocyte antigens since we have observed sera obtained from a variety of multiparous, transfused, bone marrow transplanted, and immunoneutropenic patients that react with both types of cells. Serum AYD is an example in which both the granulocyte and monocyte activity may be absorbed by either cell type from positive donors but was not absorbed by platelets, lymphoid cell lines, or chronic lymphatic leukemia cells (27). These absorptions exclude the possibility of shared monocyte-B lymphocyte antigens. Recently, we have also demonstrated that this serum reacts with cord endothelial cells, raising the question as to whether the non-B cell monocyte-endothelial antigens described by Moraes and Stastny (28-30) may also be expressed on granulocytes.

What, if any, relationship do the antigens described herein bear to the preliminary human granulocyte specificities reported by Hasegawa et al. (31)? Although we were able to compare five sera kindly provided by that group, they appeared to be either broadly reactive or completely inactive in the fluorochromatic microgranulocytotoxic assay and no correlations were evident. Subsequently, Drew et al. (11) have reported that many of their sera contained autoreactive cold antibodies, making the earlier assignments of granulocyte specificities somewhat less certain.

Finally, the question arises as to what significance these antigens may have in disease or as histocompatibility determinants. These studies were not designed to address their functional importance but the sources of the antisera detecting them point to a role in both immunoneutropenia and in granulocyte compatibility. Recently, Claas et al. (32) have reported the development of monocyte antibodies in recipients of sib-sib HLA identical bone marrow transplants and correlated their occurrence with a higher incidence of graft rejection. Because monocyte reactions were detected by the two-color fluorescent method and granulocytes were not tested, these are being extended to include granulocyte, monocyte, and lymphocyte cytotoxicity testing.

\section{ACKNOWLEDGMENTS}

Dr. J. S. Thompson was supported in part by a Senior International Fellowship of the Fogarty International Center, and N01-HB-6-2973 from the National Heart, Lung and Blood Institute of the National Institutes of Health. Dr. Claas was supported in part by the J. A. Cohen Institute for Radio- 
pathology and Radiation Protection, the Netherlands. Dr. 'Amaro was supported by the Dutch Organization for Pure Research (ZWO).

\section{REFERENCES}

1. Thorsby, E. 1969. HLA antigens on human granulocytes studied with iso-antisera obtained by skin grafting. Scand. J. Haematol. 6: 119-127.

2. Cook, K. M. 1974. Distribution of HLA antigens on blood cells. Tissue Antigens. 4: 202-209.

3. Higby, D. J., D. Bumett, K. Ruppert, E. S. Hendersen, and E. Cohen. 1977. Granulocyte transfusions: experience at Rosewell Park Memorial Institute. Prog. Clin. Biol. Res. 13: 29.3-304.

4. McCullough, J., N. Wood, B. J. Werblen, I. E. Fortuny, and E. J. Yunis. 1977. The role of histocompatibility testing in granulocyte transfusions. Prog. Clin. Biol. Res. 13: $321-327$.

5. Goldstein, I. M., H. J. Eyre, P. I. Terasaki, E. S. Henderson, and R. G. Graw. 1971. Leukocyte transfusion: role of leukocyte alloantigens in determining transfusion response. Transfusion (Phila.). 11: 19-24.

6. I alezari, P., and G. E. Bernard. 1966. An isologous antigen-antibody reaction with human neutrophils, related to neonatal neutropenia. J. Clin. Invest. 45: 1741-1750.

7. Lalezari, P., G. B. Murphy, and F. H. Allen, Jr. 1971. $\mathrm{NB}_{1}$, a new neutrophil-specific antigen involved in the pathogenesis of neonatal neutropenia. J. Clin. Invest. 50: $1108-1115$.

8. van Rood, J. J., A. van Leevwen, A. M. J. Schippers, W. H. Vooys, E. Frederiks, H. Balner, and J. G. Eernise. 1965. Leukocyte groups, the normal lymphocyte transfer test and homograft sensitivity. In Histocompatibility Testing 1965. H. Balner, F. J. Cleton, and J. G. Eernise, editors. Munksgatard International Booksellers and Publishers, Ltd., Copenhagen. 37-50.

9. Verheught, F. W. A., A. E. G. van der Borne, J. C. van Noord-Bokhorst, L. E. Nijenhuis, and C. P. Englejaiet. 1978. NI ${ }_{1}$, a new neutrophil granulocyte antigen. Vox Sang. 35: 13-17.

10. Claas, F. H. J., J. Langerak, L. J. M. Sabbe, and J. J. van Rood. 1979. $\mathrm{NE}_{1}$, a new neutrophil specific antigen. Tissue Antigens. 13: 129-134.

11. Drew, S. I., and P. I. Terasaki. 1978. Autoimmune cytotoxic granulocyte antibodies in normal persons and various diseases. Blood. 52: 941-952.

12. Thompson, J. S., J. M. Herbick, C. P. Burns, R. G. Strauss, J. W. Blaschke, J. A. Koepke, L. G. Maquire, and M. M. Goedken. 1978. Granulocyte antigens detected by cytotoxicity (GCY) and capillary agglutination (CAN). Transplant. Proc. 10: 885-888.

13. Thompson, J. S., J. M. Herbick, C. P. Bums, R. G. Strauss, J. W. Blaschke, J. A. Koepke, L. C. Maquire, and M. M. Goedken. 1979. Granulocyte-specific antigens detected by microgranulocytotoxicity. Transplant. Proc. 11: 431433.

14. Blaschke, J., C. D. Severson, N. E. Goeken, and J. S. Thompson. 1977. Microgranulocytotoxicity. J. Lab. Clin. Med. 90: 249-258.

15. Rinehart, J. J., B. J. Gormus, P. Lange, and M. E. Kaplan.
1978. A new method for isolation of human monocytes. J. Immunol. Methods. 23: 207-212.

16. Wilcox, M. B., D. W. Golde, and M. J. Cline. 1976. Cytochemical reactions of hematopoetic cells in liquid culture. J. Histochem. Cytochem. 24: 979-98.3.

17. Sakane, T., A. D. Steinberg, J. P. Reeves, and I. Greene. 1979. Studies of immune functions of patients with systemic lupus erythematosus. Complement-dependent immunoglobulin $\mathbf{M}$ anti-thymus-derived cell antibodies preferentially inactivate supressor cells. J. Clin. Invest. 63: $954-965$.

18. van Leeuwen, A., J. G. Eernise, and J. J. van Rood. 1964. A new leukocyte group with two alleles: leukocyte group five. Vox Sang. 9: 431-446.

19. Nijenhia, L. E. 1977. Genetic considerations on association between HLA and disease. Hum. Genet. 38: 175- 182.

20. Pearson, K. 19(0). On the criterion that a given system of deviations from the probable in the case of correlated system of variables is such that it can be reasonably supposed to have arisen from random sampling. Phil. Mag. (Series 5.) 50: 157-160).

21. Engelfriet, C. P. 1965. Cytotoxic antibodies against leukocytes. In Histocompatibility Testing 1965. H. Balner, F. J. Cleton, and J. G. Eernise, editors. The Williams \& Wilkins Company, Baltimore. 245-250.

22. Thorsby, E. 1967. Cell specific and common antigens of human gramulocytes and lymphocytes demonstrated with cytotoxic hetero-antibodies. Vox Sang. 13: 194-205.

23. Hasegawa, T., R. G. Graw, and P. I. Terasaki. 1973. A microgranulocyte cytotoxicity test. Transplantation (Baltimore). 15: 492-498.

24. Drew, S. I., O. J. Bergh, and J. D. McClelland. 1977. Antigenic specificities detected on papainized human granulocytes by microgranulocytotoxicity. Transplant. Proc. 9: 6.39-645.

25. Thompson, J. S., J. Blaschke, S. Birney, and C. D. Severson. 1977. Detection of allospecific granulocyte antigens by capillary agglutination and microgranulocytotoxicity. Transplant. Proc. 9: 1895-1897.

26. Thompson, J. S. 1976. In Manual of Clinical Immunology. E. R. Rose and A. Friedman, editors. American Society of Microbiology, Washington, D. C. 868-873.

27. Thompson, J. S., C. D. Severson, N. A. Greazel, and S. Ferrone. 1977. Detection of HLA (Ia) and non-HLA specificities by "B-Cell" Antisera. Transplant. Proc. 9: 597-599.

28. Moraes, J. R., and P. Stastny. 1977. Human Endothelial Cell Antigens: Molecular independence from HLA and expression in blood monocytes. Transplant. Proc. 9: 605-607.

29. Moraes, J. R., and P. Stastny. 1977. New antigen system expressed on human endothelial cells. J. Clin. Invest. 60: $449-454$.

30. Stastny, Pl. 1978. Endothelial monocyte antigens in man. Transplant. Proc. 10: 875-877.

31. Hasegawa, T., O. J. Bergh, M. R. Mickey, and P. I. Terasaki. 1975. Preliminary human granulocyte specificities. Transplant. Proc. 7: 75-80.

32. Claas, F. J. J., J. J. van Rood, R. P. Warren, P. L. Weiden, P. J. Su, and R. Storb. 1979. The detection of non-HLA antibodies and their possible role in bone marrow graft rejection. Transplant. Proc. 11: 423-426. 